\title{
Exosomes Transport Anti-Human Immunodeficiency Virus Factors from Human Cervical Epithelial Cells to Macrophages
}

\author{
Xi-Qiu Xu Biao Zhang Le Guo Yu Liu Feng-Zhen Meng Xu Wang \\ Wen-Hui Hu Adil I. Khan Wen-Zhe Ho \\ Department of Pathology and Laboratory Medicine, Temple University Lewis Katz School of Medicine, Philadelphia, \\ PA, USA
}

\author{
Keywords \\ Human cervical epithelial cells · Exosomes · Toll-like \\ receptor $3 \cdot$ Macrophages · IFN-Stimulated genes · Human \\ immunodeficiency virus
}

\begin{abstract}
The female reproductive tract (FRT) is a major site of HIV sexual transmission. As the outermost layer of cells in the FRT, the human cervical epithelial cells (HCEs) have direct contact with HIV or infected cells. Our early work showed that supernatant (SN) from TLR3-activated HCEs contain the antiviral factors that could potently inhibit HIV replication in macrophages. However, it remains to be determined how HCEs transport the anti-HIV factors to macrophages. This followup study examined the role of exosomes in HCE-mediated anti-HIV activity. We found that TLR3 activation of HCEs resulted in the release of exosomes that contained multiple IFN-stimulated genes (ISGs: ISG56, OAS1, MxA, and Mx2) and the HIV restriction microRNAs (miR-28, miR-29 family members, miR-125b, miR-150, miR-382, miR-223, miR-20a, and miR-198). The depletion of exosomes from SN of TLR3-activated HCEs diminished HCE-mediated anti-HIV activity in macrophages, indicating that HCE-derived exosomes are responsible for transporting the antiviral molecules to macrophages. These in vitro findings suggest a novel antiviral
\end{abstract}

karger@karger.com www.karger.com/jin

Karger"

GOPEN ACCESS
(C) 2021 The Author(s).

Published by S. Karger AG, Basel

This is an Open Access article licensed under the Creative Commons Attribution-NonCommercial-4.0 International License (CC BY-NC) (http://www.karger.com/Services/OpenAccessLicense), applicable to the online version of the article only. Usage and distribution for commercial purposes requires written permission. mechanism by which HCEs participate in the FRT innate immunity against HIV infection. Further in vivo studies are necessary in order to develop an exosome-based delivery system for prevention and treatment of HIV infection through sexual transmission.

(c) 2021 The Author(s).

Published by S. Karger AG, Basel

\section{Introduction}

Human immunodeficiency virus (HIV) transmission to women through the female reproductive tract (FRT) accounts for about $40 \%$ of all HIV transmission events [1]. During sexual intercourse, HIV in male semen can pass the female genital epithelium $[2,3]$ and establish infection in the target cells of the underlying lamina propria, including $\mathrm{CD} 4^{+} \mathrm{T}$ cells, dendritic cells, and macrophages $[4,5]$. As the first layer cells in the FRT, the human cervical epithelial cells (HCEs) are in the direct contact with HIV or infected cells. Therefore, to understand an important role of HCEs in the FRT innate immunity against viral infection is of significance and interest [6]. Several independent studies [7-9] have shown that HCEs have the immune protection function to identify pathogen-associated molecular patterns on pathogens through pattern recognition receptors. We have documented that the HCEs 
possess a functional toll-like receptor 3/interferon (TLR3/ IFN) signaling system [10], which could be activated by a TLR3 ligand, inducing the production of the antiviral factors that suppressed HIV replication in primary human macrophages [11]. These earlier studies, however, did not address the question of how HCE-released anti-HIV factors, particularly the IFN-stimulated genes and the HIV restriction miRNAs, are transported to macrophages.

There has been a rapid growth in investigating the role of the extracellular vehicles (EVs), including exosomes, ectosomes, oncosomes, microvesicles, apoptotic bodies, microparticles, and other EV subsets in cell-cell communications [12]. Among them, exosomes (EV s of endosomal origin) play a crucial role in the intercellular transportation [13-15]. Exosomes are formed through the fusion of multivesicular bodies (MVBs) with the host cell plasma membrane and then release of intraluminal vesicles (ILVs) as exosomes [16]. Specifically, exosomes originate in endosomal compartments called MVBs, which are late endosomes containing multiple ILVs formed by the invagination of the endosomal membrane [17]. When MVBs fuse with the plasma membrane, ILVs are released as exosomes. Differential ultracentrifugation is the most commonly used exosome separation. Isolated exosomes have a typical cup-shaped structure, with a diameter of 30-100 $\mathrm{nm}$ and are rich in endosome-associated proteins [18]. The most common exosome-carried proteins can act as specific markers, including CD63, CD81, Alix (apoptosislinked gene 2-interacting protein $\mathrm{x}$ ), LAMP2 (lysosomeassociated membrane protein 2), TSG101 (tumor susceptibility gene 101 protein), and PECAM-1 (platelet and endothelial cell adhesion molecule-1) [19, 20]. In addition, the identity and purity of exosome can be determined by lacking of the cellular markers such as EEA1 (early endosomal antigen 1 , an endosomal marker), cytochrome C (a mitochondrial marker), and GAPDH (cytoskeleton marker) [21]. In addition to exosome-associated proteins, exosomes carry various molecules in their lumen, including lipids, proteins, mRNAs, microRNAs (miRNAs), and long noncoding RNAs (lncRNAs) [22]. After their release into the extracellular space, exosomes can act locally or circulate through various bodily fluids, including blood and lymph, resulting in a systemic response [23]. The recent studies have shown that exosome-mediated intercellular communication also plays a crucial role in the antiviral innate immunity $[21,24,25]$. We, thus, examined the role of exosomes produced by HCEs in transporting the antiviral factors to HIV-infected macrophages. We also investigated the mechanisms by which the exosomes participate in HCE-mediated innate immunity against HIV.

\section{Materials and Methods}

\section{Reagents}

Rabbit antibodies against exosome-linked markers, ISGs, and HIV p24 protein were purchased from Cell Signaling Technology (Danvers, MA, USA). PKH67 fluorescent cell linker kits and PKH26 fluorescent kit were purchased from Sigma-Aldrich Co. LLC. Exosome-depleted fetal bovine serum (FBS) was purchased from System Biosciences, Inc (Mountain View, CA, USA). All culture plasticwares were obtained from Corning (Corning, NY, USA). Unless otherwise specified, all other culture reagents were purchased from Invitrogen (San Diego, CA, USA).

\section{Cells Culture}

The HCE line (End1/E6E7 cells) has been extensively studied and well established as a HCE model $[26,27]$. The cells were cultured in keratinocyte growth medium (Gibco, Grand Island, NY, USA) supplemented with the provided recombinant epidermal growth factor $(0.1 \mathrm{ng} / \mathrm{mL})$ and bovine pituitary extract $(50 \mu \mathrm{g} / \mathrm{mL})$. Monocytes were obtained from Human Immunology Core at the University of Pennsylvania (Philadelphia, PA). The core has the institutional review board approval for blood collection from healthy donors. Monocytes isolated from peripheral blood differentiated into macrophages as described [28, 29]. Briefly, freshly isolated monocytes were cultured in a 48 -well plate $\left(2.5 \times 10^{5}\right.$ cells/ well) in complete DMEM containing $10 \%$ FBS and 1\% penicillinstreptomycin without any growth factors. Monocyte-derived macrophages refer to monocytes cultured in vitro for 7 days.

\section{Exosome Isolation}

HCES (End1/E6E7 cells) were transfected with Poly I:C for $4 \mathrm{~h}$ and cultured in fresh-culturing medium containing $10 \%$ exosomefree FBS. At 48 h post-transfection, End1/E6E7 cell supernatant (SN) was collected and exosomes were isolated by the differential centrifugation as described [24]. Briefly, SN was centrifuged at $300 \mathrm{~g}$ for $10 \mathrm{~min}, 2,000 \mathrm{~g}$ for $10 \mathrm{~min}, 10,000 \mathrm{~g}$ for $30 \mathrm{~min}$, and $100,000 \mathrm{~g}$ for $70 \mathrm{~min}$. For further purification, the pellets were washed with PBS and centrifuged at $100,000 \mathrm{~g}$ for $70 \mathrm{~min}$. The pellets were then resuspended in $100 \mu \mathrm{L}$ PBS and freshly used.

\section{Electron Microscopy of Isolated Exosomes}

Isolated exosomes from HCE SN were resuspended in $10 \mu \mathrm{L}$ PBS and spotted onto Formvar-coated grids (200 mesh). Adsorbed exosomes were then fixed in $2 \%$ ( $\mathrm{vol} / \mathrm{vol}$ ) paraformaldehyde at room temperature for $5 \mathrm{~min}$. After fixation, the exosomes were negatively stained with uranyl acetate. The grids were observed under the electron microscope (CM100; Philips, Amsterdam, the Netherlands).

\section{Labeling Exosomes with Fluorescent}

Macrophages were cultured at a density of $2.5 \times 10^{5}$ cells/well in 48-well plates. Isolated exosomes from SN of HCE cultures were labeled with PKH67 fluorescent according to the manufacturer's protocol (Sigma-Aldrich). Purified PKH67 exosomes were incubated with macrophages and cultured at $37^{\circ} \mathrm{C}$ for $18 \mathrm{~h}$ in a $\mathrm{CO}_{2}$ incubator. Macrophages were then stained with a PKH26 fluorescent for membrane and Hoechst 33342 for nuclei and then washed 3 times with $1 \times$ PBS. The cells were photographed under a confocal microscope (Nikon A1R, Nikon, Japan). 


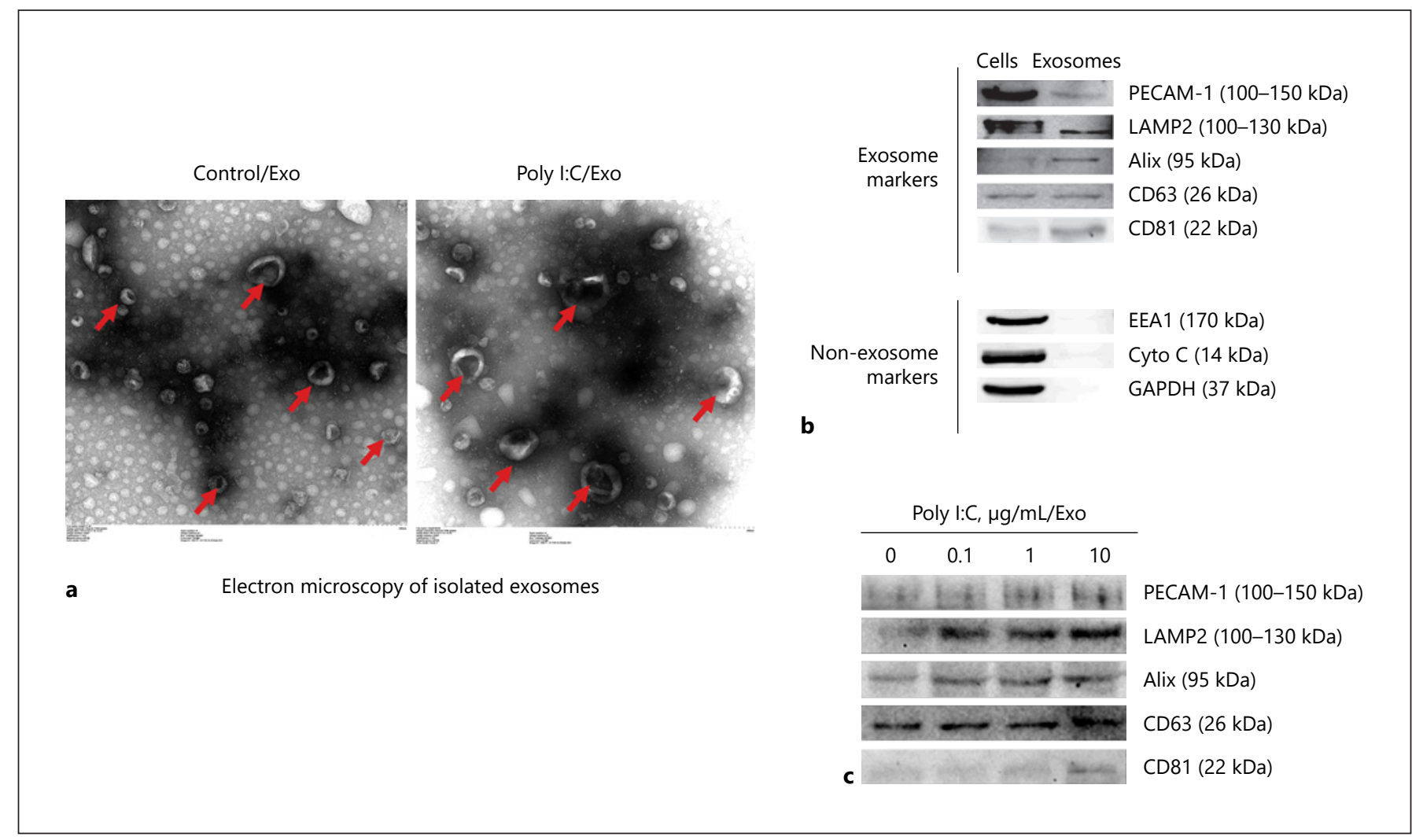

Fig. 1. Characterization of exosomes of End1/E6E7 cells. a Electronic microscope observation of End1/E6E7 cell-derived exosomes. End1/E6E7 cells were transfected with or without Poly I:C $(1 \mu \mathrm{g} / \mathrm{mL})$ for $48 \mathrm{~h}$, and cell culture $\mathrm{SN}$ was collected for exosome isolation. Isolated exosomes were then fixed in $2 \%(\mathrm{v} / \mathrm{v})$ paraformaldehyde. The fixed exosomes were negatively stained with uranyl acetate and then observed by electron microscope. b Western blot assay of exosome markers and nonexosome markers. End1/ E6E7 cells were cultured in exosome-free culture media for $48 \mathrm{~h}$ in a 48-well culture plate. Total proteins from End1/E6E7 cells and

Real-Time PCR Quantification of $m R N A$ and microRNA

Total RNA from cultured cells was extracted with Tri-Reagent (Molecular Research Center, OH, USA) as previously described [10]. Total RNA $(1 \mu \mathrm{g})$ was subjected to reverse transcription using reagents from Promega (Promega, WI, USA). The resulting cDNA was then used as a template for qPCR quantification. The real-time PCR for the quantification of GAPDH, HIV gag, and ISG mRNA was performed with IQ SYBR Green Supermix (Bio-Rad Laboratories, Hercules, CA, USA) as described previously [30]. The levels of GAPDH mRNA were used as an endogenous reference to normalize the quantities of the tested mRNAs. The primers were synthesized by Invitrogen Inc., and the sequences of oligonucleotide primers are shown in Table 1. MicroRNA was extracted with the miRNeasy Mini Kit (Qiagen, Valencia, CA, USA) based on the manufacturer's instructions. RNA was reverse transcribed with the miScript Reverse Transcription Kit (Qiagen). Reverse transcription PCR for the quantification of a subset of miRNAs was carried out with the isolated exosomes were extracted for Western blot analysis. The expression of the specific exosomal markers (Alix, CD63, CD81, PECAM-1, and LAMP2) and nonexosomal markers (EEA1, cytochrome $\mathrm{C}$, and GAPDH) are indicated. c The presence of exosome markers in End1/E6E7 cell-derived exosomes upon Poly I:C transfection. End1/E6E7 cells were transfected with or without Poly I:C $(0.1,1,10 \mu \mathrm{g} / \mathrm{mL})$ for $48 \mathrm{~h}$. Cell culture SN was collected for exosome isolation and to detect the expression of the specific exosomal markers (Alix, CD63, CD81, PECAM-1, and LAMP2). $\mathrm{SN}$, supernatant.

miScript Primer Assays and with the miScript SYBR Green PCR Kit from Qiagen as previously described [31]. Synthetic Caenorhabditis elegans miRNA-39 (cel-miR-39) was used as a spiked-in miRNA for normalization.

\section{Western Blotting}

Total cell lysates were prepared by the cell extraction buffer (Thermo Fisher Scientific, MA, USA) with $1 \%$ protease inhibitor cocktail (Sigma, MO, USA) according to the manufacturer's instructions. Equal amounts of protein lysates $(30 \mu \mathrm{g})$ were separated on $4-12 \%$ sodium dodecyl sulfate polyacrylamide gel electrophoresis precast gels and transferred to an Immunobiolon-P membrane (Millipore, Germany). The blots were incubated with primary antibodies in 5\% nonfat milk in PBS with $0.05 \%$ Tween 20 overnight at $4^{\circ} \mathrm{C}(O A S 1,1: 1,000 ;$ OAS2, 1:1,000; ISG15, 1:1,000; ISG56, 1:1,000; MxA, 1:1,000; Mx2, 1:1,000; GBP-5, 1:1,000; Alix, 1:1,000; LAMP2, 1:1,000; CD63, 1:1,000; CD81, 1:1,000; cytochrome C, 1:1,000; PECAM-1, 1:1,000; EEA1, 1:1,000; GAPDH, 


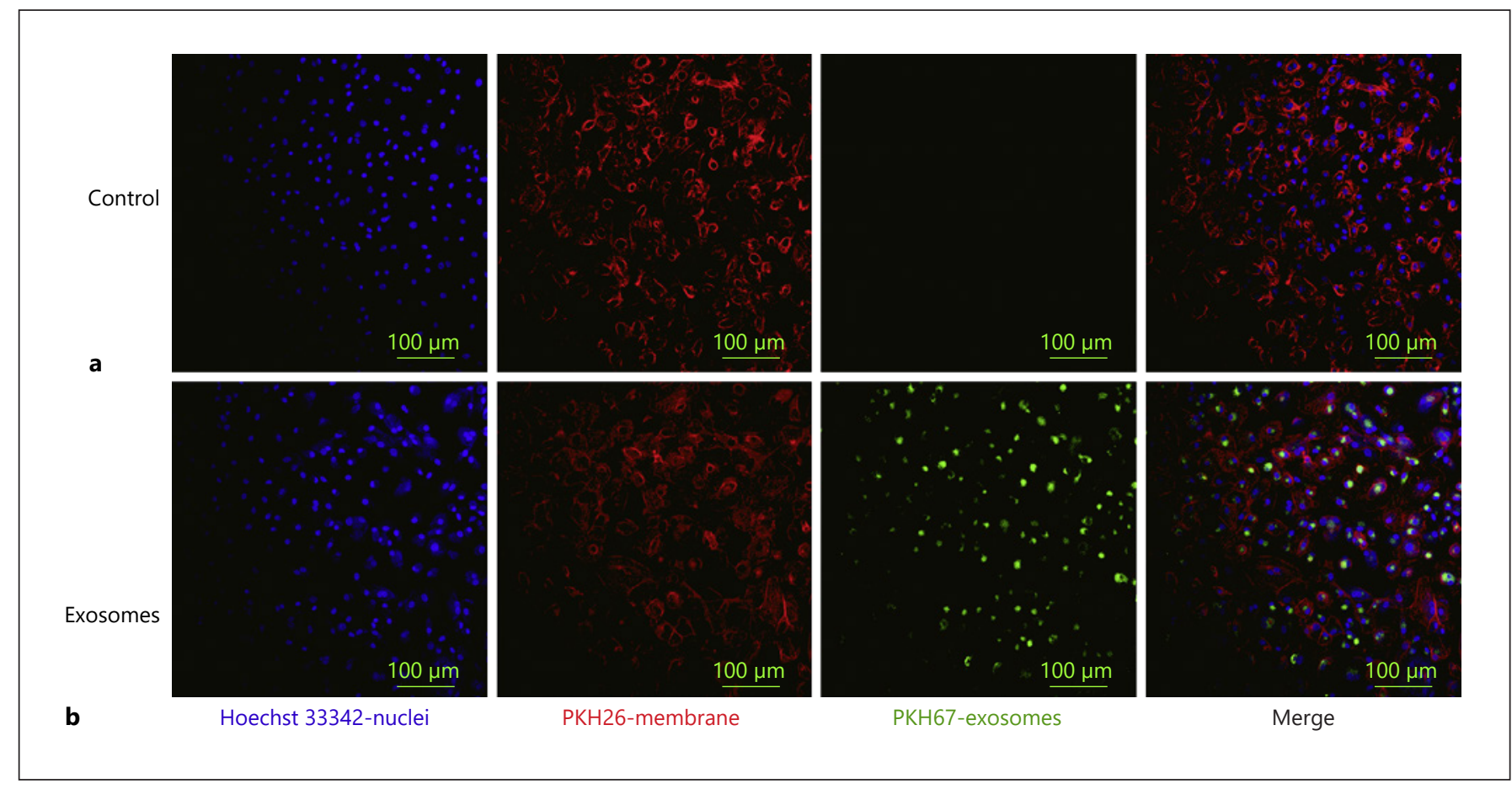

Fig. 2. TLR3 signaling of End1/E6E7 cells released exosomes can be taken up by the macrophages. End1/E6E7 cells were cultured in exosome-free culture media for $48 \mathrm{~h}$. Isolated exosomes were labeled with PKH67 fluorescent cell linker (green) and then added to Exosome Spin Columns. Purified PKH67-labeled exosomes were incubated with macrophages for $48 \mathrm{~h}$. Macrophages were then stained with Hoechst 33342 (blue) for nuclei and PKH26 fluorescent cell linker (red) and then observed under a fluorescence microscope (original magnification, $\times 100$ ).

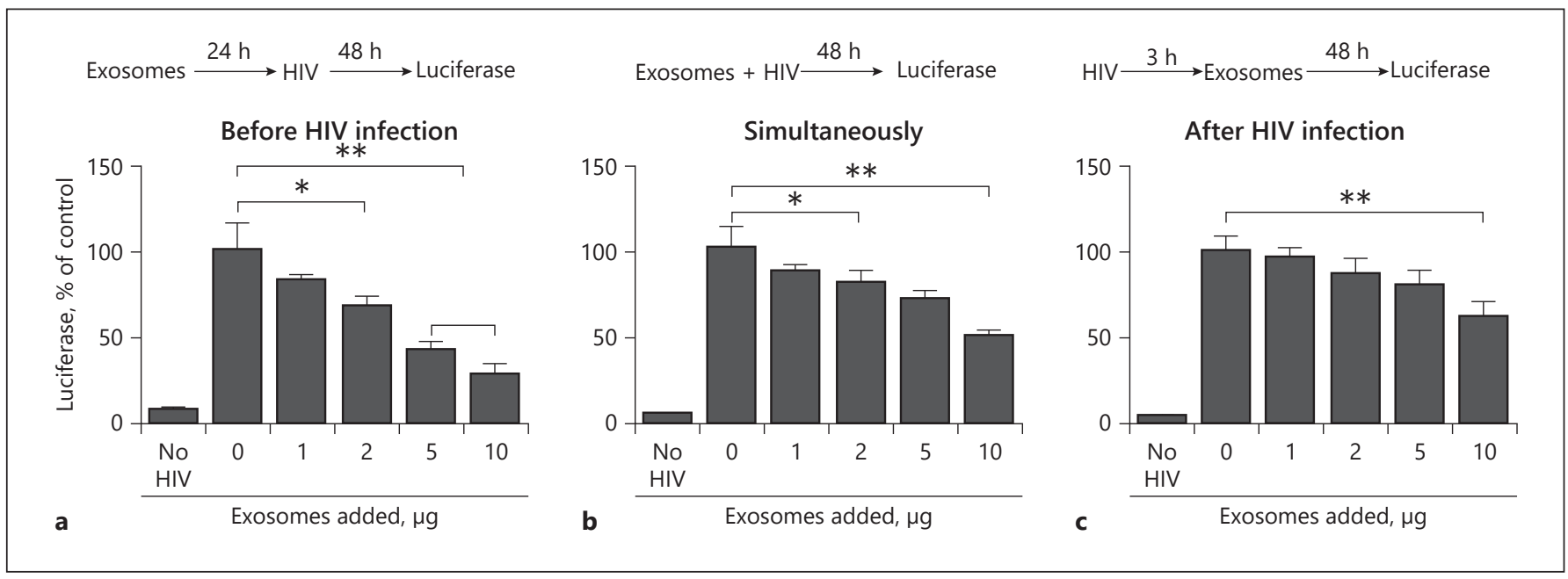

Fig. 3. Effect of exosomes from Poly I:C-stimulated End1/E6E7 cell cultures on HIV infection of TZM-bl cells. TZM-bl cells were treated with or without exosomes (1, 2, 5, and $10 \mu \mathrm{g})$ from End1/ E6E7 cell cultures stimulated with Poly I:C for $24 \mathrm{~h}$ prior to (a), simultaneously (b), or after HIV infection (c). Luciferase activity of TZM-bl cells was measured at $48 \mathrm{~h}$ post-HIV infection. The results are the mean \pm SD of triplicate cultures, representative of 3 experiments $\left({ }^{*} p<0.05\right.$ and $\left.{ }^{* *} p<0.01\right)$. HIV, human immunodeficiency virus. 


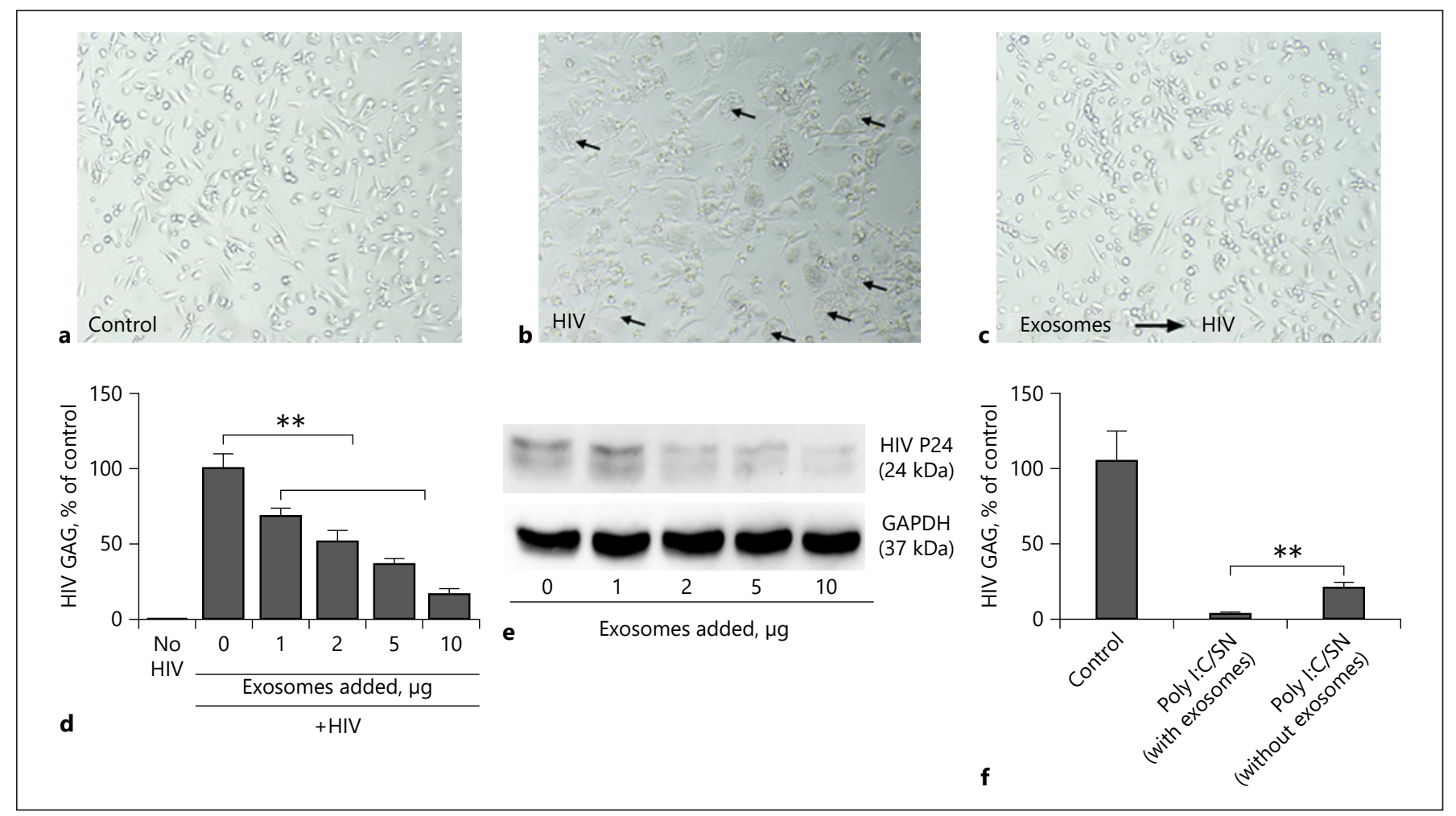

Fig. 4. End1/E6E7 cell-derived exosomes inhibit HIV replication in macrophages. End1/E6E7 cells were transfected with or without Poly I:C $(1 \mu \mathrm{g} / \mathrm{mL})$ for $48 \mathrm{~h}$, and then, cell culture $\mathrm{SN}$ was collected for exosome isolation. a-c Morphologic observations of uninfected and HIV Bal-infected macrophages with or without exosomes pretreatment (arrows indicate syncytium, magnification $\times 100$ ). d Macrophages were pretreated for $24 \mathrm{~h}$ with or without End1/ E6E7 cell-derived exosomes (1, 2, 5, and $10 \mu \mathrm{g})$ and then infected with HIV Bal. Total RNA extracted from macrophages was sub-

1:1,000; and HIV p24, 1:1,000). Horseradish peroxidase-conjugated appropriate second antibodies were diluted 1:2,000 to 1:8,000 in $5 \%$ nonfat milk PBS with $0.05 \%$ Tween 20 . Blots were developed with SuperSignal West Pico Chemiluminescent Substrate (Thermo Fisher Scientific, MA, USA).

\section{TZM-bl Assay}

The anti-HIV activity was assessed with the TZM-bl, a commonly used cell line for evaluating the efficacy of neutralizing antibodies and small molecular inhibitors in HIV research [32]. TZM-bl cells contain the reporter gene (firefly luciferase) for the quantitative detection of HIV Tat-dependent luciferase activities after HIV infection. The cells were prepared and maintained as previously described.

End1/E6E7 Cell-Derived Exosomes Treatment and HIV Infection

TZM-bl cells were seeded in a 96-well microtiter plate $\left(10^{4}\right.$ cells/ well) and allowed to achieve confluence overnight at $37^{\circ} \mathrm{C}$. The cells were then treated with or without End1/E6E7 cell-derived exosomes for $24 \mathrm{~h}$ prior to, simultaneously, or post-HIV (Bal strain) infection. jected to real-time PCR for HIV gag gene quantification 8 days post-HIV infection. e The intracellular p24 protein level was measure by Western blot. $\mathbf{f}$ The inhibition of HIV replication by End1/ E6E7 cell SN with or without exosome depletion. The data were expressed as HIV gag levels relative (\%) to control (without treatment, which is defined as $100 \%$ ). The results are the mean \pm SD of triplicate cultures, representative of 3 experiments $\left({ }^{* *} p<0.01\right)$. SN, supernatant; HIV, human immunodeficiency virus.

Luciferase activity of TZM-bl cells was measured at $48 \mathrm{~h}$ post-HIV infection. Macrophages were treated with or without End1/E6E7 cell-derived exosomes for $24 \mathrm{~h}$ and then infected with HIV Bal strain. At day 8 postinfection, the cells were collected for HIV gag gene expression. TZM-bl cells and primary human macrophages were infected with an equal amount (p24 $30 \mathrm{ng} / 10^{6}$ cells) of cell-free HIV Bal for $3 \mathrm{~h}$ at $37^{\circ} \mathrm{C}$ in the presence or absence of End1/E6E7 cell-derived exosomes. To deplete exosomes, the SN from Poly I:C-transfected HCEs was incubated with anti-CD63 antibody-conjugated with Dynabeads overnight at $4^{\circ} \mathrm{C}$ and then separated in a magnetic field.

\section{Data Analysis}

When appropriate, data were obtained from at least 3 independent experiments and expressed as mean \pm SD. For comparison of the mean of 2 groups, the statistical significance was measured by Student's $t$ test. To compare the difference between multiple groups, statistical significance was analyzed using ANOVA followed by Tukey's test. Calculations were performed with GraphPad Prism statistical software (GraphPad Software Inc., San Diego, CA, USA). Statistical significance was defined as $p<0.05$. 


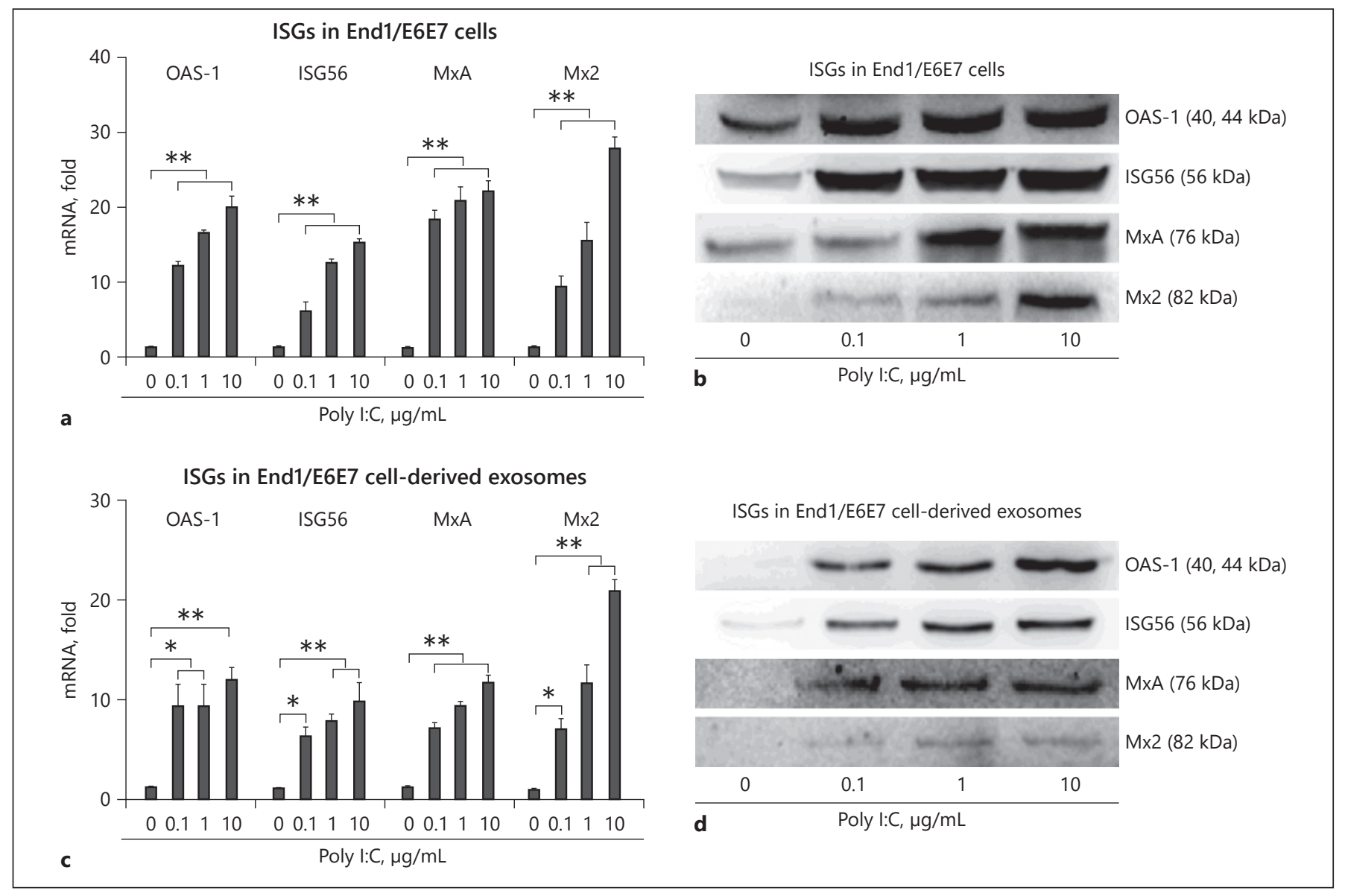

Fig. 5. Characterization of the antiviral factors in the exosomes of TLR3-stimulated End1/E6E7 cells. End1/E6E7 cells were transfected with or without Poly I:C $(0.1,1$, and $10 \mu \mathrm{g} / \mathrm{mL})$ and then cells and exosomes were collected to extract RNA and proteins. a, c Cellular RNAs $(1 \mu \mathrm{g})$ and exosomal RNAs $(1 \mu \mathrm{g})$ were subjected to real-time PCR for indicated genes (OAS1, ISG56, MxA,

\section{Results}

\section{HCEs Produce Exosomes}

To determine the role of exosomes in intercellular communications between HCEs and macrophages, we examined whether TLR3 signaling of End1/E6E7 cells can induce the production of exosomes. As shown in Figure 1a, electronic microscopy analysis of the purified pellets observed small membrane vesicles of $30-100 \mathrm{~nm}$ in diameter, which have a typical cup-shaped exosome structure. As shown in Figure 1b, Poly I:C-transfected cells produced exosomes that had the special exosomal markers (Alix, CD63, CD81, LAMP2, and PECAM-1) and lacked the cellular markers (EEA1, cytochrome $\mathrm{C}$, and $G A P D H)$. Because TLR3 localizes in the endosomal com-

and $M \times 2$ ) and GAPDH RNA. Data are expressed as indicated gene RNA levels relative (fold) to untreated control, which is defined as 1. b, d Cellular proteins $(20 \mu \mathrm{g})$ and exosomal proteins $(20 \mu \mathrm{g})$ were subjected to Western blot assay using antibodies to OAS1, ISG56, $M x A$, and $M x 2$. The results are the mean \pm SD of triplicate cultures, representative of 3 experiments $\left({ }^{*} p<0.05\right.$ and $\left.{ }^{* *} p<0.01\right)$.

partment of the cells, the transfection of Poly I:C is necessary for activating TLR3 in the cells. As shown in Figure 1c, Poly I:C transfection could increase the expression of the special exosomal markers (Alix, CD63, CD81, LAMP2, and PECAM-1) in End1/E6E7 cell-produced exosomes.

\section{End1/E6E7 Cell-Derived Exosomes Can Be Taken Up by Macrophages}

To visualize whether exosomes isolated from End1/ E6E7 cell culture SN could be delivered into macrophages, macrophages were incubated with exosomes labeled with fluorescent dye PKH67 (green) for $18 \mathrm{~h}$ in a $\mathrm{CO}_{2}$ incubator. Macrophages were then stained with a PKH26 (red) for membrane and Hoechst 33342 (blue) for nuclei. The cells were photographed under a confocal microscope 


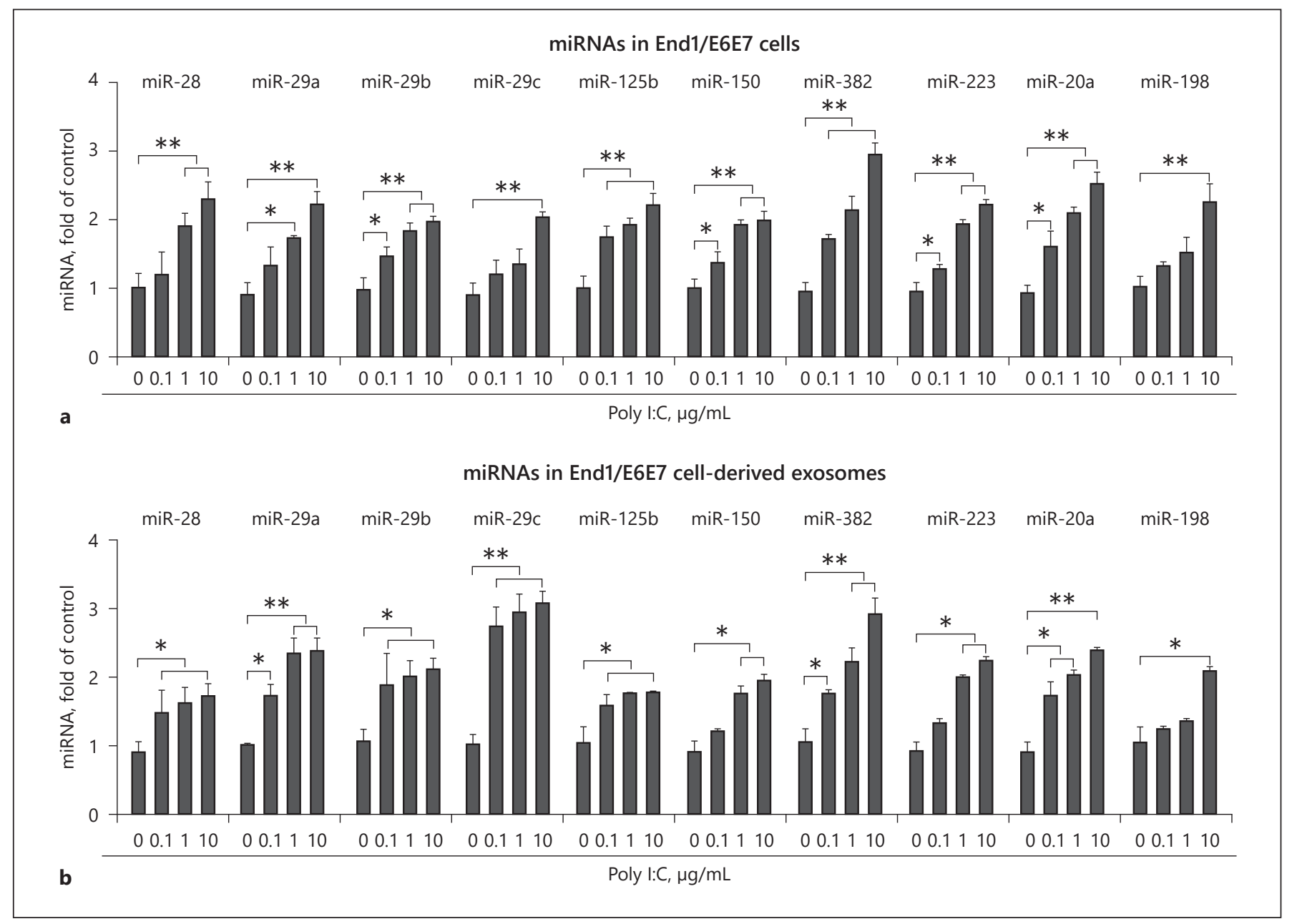

Fig. 6. Characterization of the microRNAs in the exosomes of TLR3-stimulated. End1/E6E7 cells cultured in exosome-free media were transfected with or without Poly I:C $(0.1,1$, and $10 \mu \mathrm{g} /$ $\mathrm{mL}$ ) for $48 \mathrm{~h}$. Cell culture SN was collected for exosome isolation. End1/E6E7 cells (a) and isolated exosomes (b) were subjected for RNA extraction. Total RNA $(1 \mu \mathrm{g})$ was then subjected to real-time

(Nikon A1R, Nikon, Japan). As shown in Figure 2, PKH67labeled exosomes were observed within macrophages.

\section{End1/E6E7 Cell-Derived Exosomes Inhibit HIV}

\section{Infection in TZM-bl Cells}

We first examined whether End1/E6E7 cell-derived exosomes can suppress HIV replication in TZM-bl cells. TZM-bl cells were treated with or without End1/E6E7 cell-derived exosomes for $24 \mathrm{~h}$ prior to, simultaneously, or post-HIV (Bal strain) infection. Luciferase activity of TZM-bl cells was measured at $48 \mathrm{~h}$ post-HIV infection. HIV infection in TZM-bl cells was positively correlated with the luciferase activity in the cells. As shown in Figure
RT-PCR to quantify the levels of miR-28, miR-29 family members, miR-125b, miR-150, miR-382, miR-223, miR-20a, and miR-198 in End1/E6E7 cells and exosomes. Synthetic Caenorhabditis elegans miRNA-39 (cel-miR-39) was used as a spiked-in miRNA for normalization. The levels of miRNAs were plotted as fold of control. $\mathrm{SN}$, supernatant. 


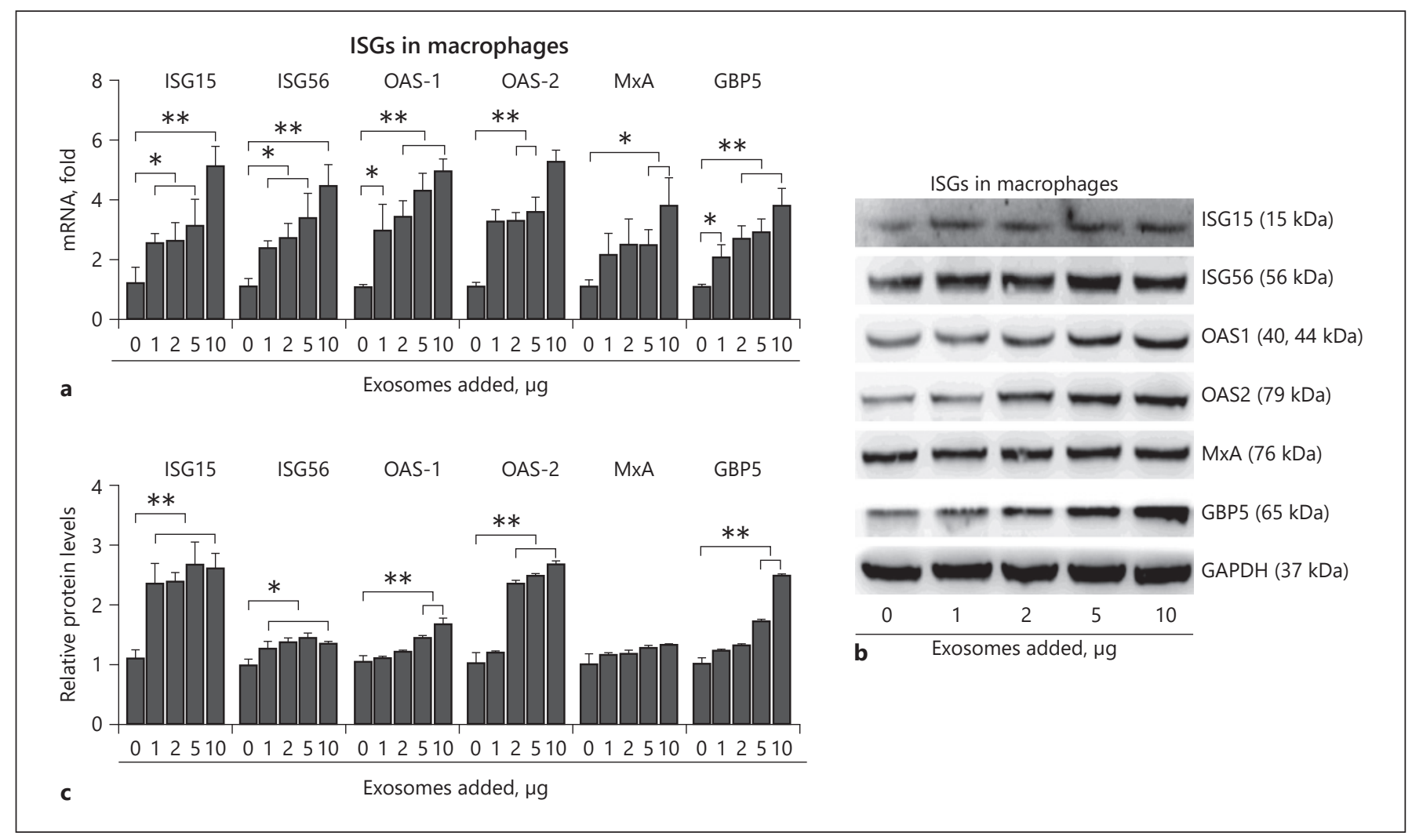

Fig. 7. Effect of End1/E6E7 cell-derived exosomes on the expression of ISGs in macrophages. Primary human macrophages were treated with exosomes from Poly I:C-stimulated End1/E6E7 cells. The mRNA (a) and protein levels ( $\mathbf{b}, \mathbf{c})$ of the ISGs were examined by qRT-PCR and Western blot, respectively. Data were obtained from 3 independent experiments and expressed as the mean \pm standard deviation $\left({ }^{*} p<0.05\right.$ and $\left.{ }^{* *} p<0.01\right)$. treatment of HIV-infected macrophages with exosomes significantly reduced virus-induced giant syncytia (Fig. 4c). In addition, HIV replication was also significantly inhibited in macrophages pretreated with End1/ E6E7 cell-derived exosomes at both genomic (Fig. 4d) and protein levels (Fig. 4e). To confirm the role of the exosomes in End1/E6E7 cell-mediated anti-HIV activity, we examined the antiviral potency of End1/E6E7 cell SN with or without exosome depletion. Figure $4 \mathrm{f}$ showed that while SN from Poly I:C-transfected End1/E6E7 cell cultures significantly suppressed HIV replication in macrophages, the depletion of exosomes from the culture SN diminished End1/E6E7 cell-mediated anti-HIV activity in macrophages.

\section{Exosomes Contain anti-HIV ISGs and miRNAs}

To determine the mechanism of the exosome-mediated anti-HIV activity, we next determined whether the exosomes carry the antiviral IFN-stimulated genes. As shown in Figure 5a, Poly I:C transfection of End1/E6E7 cells induced the mRNA expression of several key antiHIV ISGs (ISG56, OAS1, MxA, and Mx2). The expression of these ISGs was also detected in the exosomes isolated from End1/E6E7 cell cultures (Fig. 5c). In addition, we also observed an increase of the ISGs in both End1/E6E7 cells (Fig. 5b) and End1/E6E7 cell-derived exosomes (Fig. $5 \mathrm{~d}$ ) at protein levels. It is known that miRNAs can be compartmentalized in cell-released exosomes and exert biologic functions on recipient cells $[33,34]$. We, thus, examined whether the exosomes contain the anti-HIV miRNAs (miR-28, miR-29 family members, miR-125b, miR-150, miR-382, miR-223, miR-20a, and miR-198). As shown in Figure 6a and b, both End1/E6E7 cells and purified exosomes from Poly I:C-transfected End1/E6E7 cells induced the expression of the anti-HIV miRNAs.

\section{End1/E6E7 Cell-Derived Exosomes Induce ISGs in Macrophages}

We also examined the immune response in macrophages that were treated with End1/E6E7 cell-derived exo- 


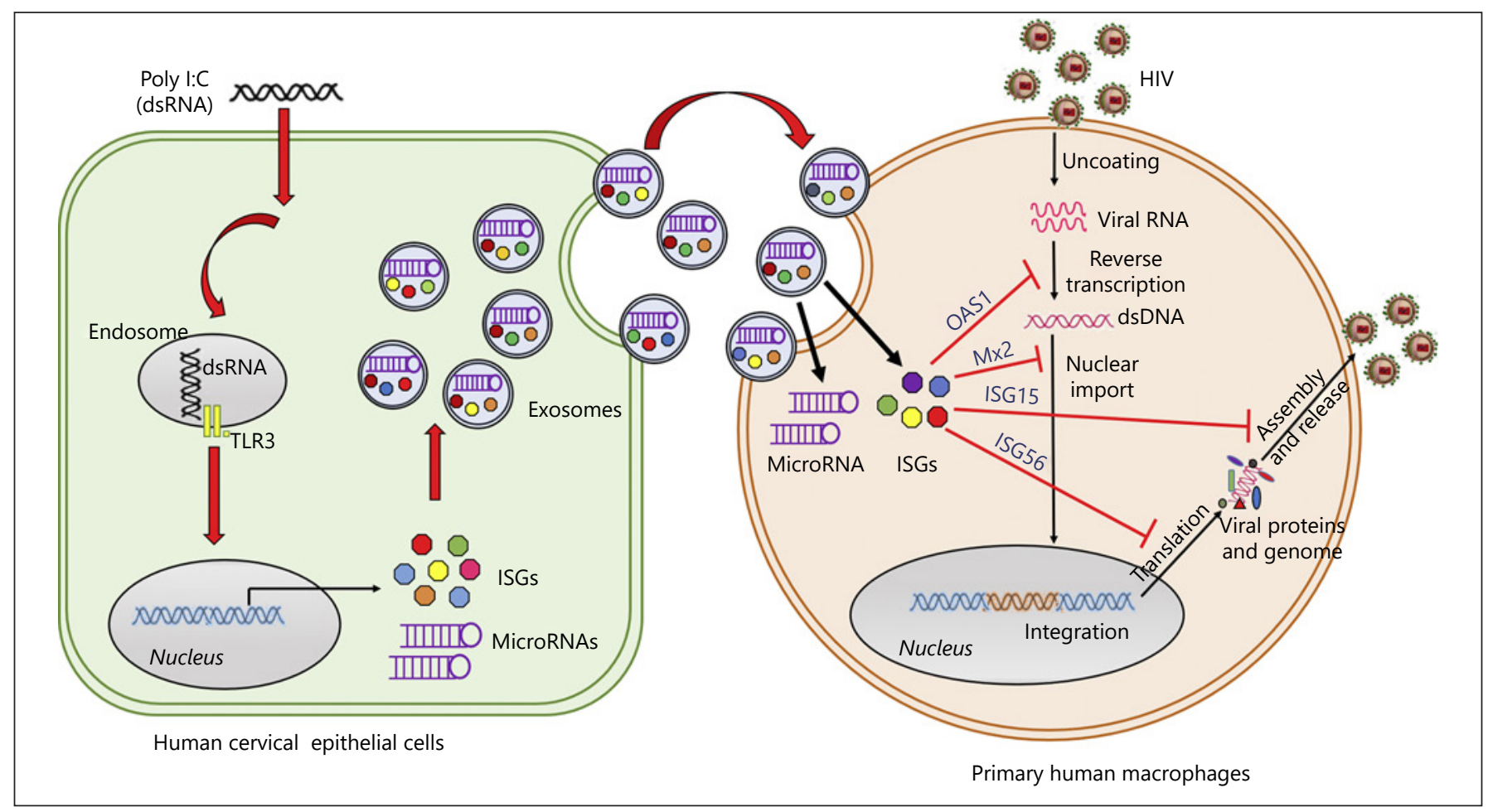

Fig. 8. Schematic diagram of mechanisms for exosome-mediated HIV inhibition in HCEs. Stimulation of HCEs with double-stranded RNA (Poly I:C) results in activation of TLR3 signaling pathway and production of the exosomes that contain the multiple cellular antiviral factors, including several key IFN-stimulated genes (ISGs: ISG56, OAS1, MxA, and Mx2) and the HIV restriction microRNAs

somes. As shown in Figure 7, treatment of macrophages with the exosomes isolated from Poly I:C-transfected End1/ E6E7 cells induced the expression of the anti-HIV ISGs at both mRNA (Fig. 7a) and protein levels (Fig. 7b, c).

\section{Discussion}

Our earlier work showed that SN from TLR3-activated HCEs contained the multiple antiviral factors that could effectively suppress HIV replication in macrophages [11]. However, the mechanisms by which HCEs confer immune protection to macrophages remain to be determined. The present study examined the role of exosomes in HCE-mediated anti-HIV activity. Exosomes are essential in the intercellular communication and play a role in host antiviral activities [21,25]. Exosomes deliver their contents to recipient cells, through which they regulate host cell functions. This transporting function of exosomes is crucial in the bio-
(miR-28, miR-29 family members, miR-125b, miR-150, miR-382, miR-223, miR-20a, and miR-198). These exosomes can be released by HCEs and taken up by HIV-infected macrophages where the released antiviral ISGs and the HIV restriction miRNAs can inhibit HIV at different steps of viral replication. HCEs, human cervical epithelial cells; HIV, human immunodeficiency virus.

logic processes, including immune response to viral infections $[35,36]$. Therefore, it is of great interest to determine whether exosomes play a role in the intercellular communication between HCEs and macrophages. We showed that HCEs produced and released exosomes that could be taken up by macrophages (Fig. 1, 2). Importantly, exosomes isolated from HCEs, when added to HIV-infected macrophages, inhibited HIV replication (Fig. 4). This inhibitory effect on HIV is likely due to the antiviral ISGs and the HIV restriction miRNAs in the exosomes as the exosomes from Poly I:C-transfected HCEs were enriched with the antiviral ISGs at both mRNA and protein levels (Fig. 5). Studies have shown that cytosolic RNA is taken up into EVs undergoing inward budding from the cell membrane, and this budding-in process is designated as RNA loading into exosomes [37]. Importantly, RNA in exosomes is protected from RNase degradation and stable under various environment conditions [38]. 
Experimental evidence indicates that several ISGs, including ISG15, ISG56, OAS1, MxA, and Mx2, possess anti-HIV properties $[39,40]$. For example, ISG15 plays a crucial role in the late stages of HIV assembly and release [41, 42]; OAS1 suppresses HIV by interfering with the viral RNA reverse transcription [43]; $M \times 2$ can reduce HIV replication as it has the ability to inhibit the capsid-dependent nuclear import of subviral complexes [44]. In addition to the ISGs, we also determined whether exosomes isolated from HCEs contain the HIV restriction miRNAs. Studies have shown that miRNAs can be packaged into and carried by exosomes at the immune synapse, regulating gene expression of the recipient cells [45, 46]. We demonstrated that both End1/E6E7 cells and End1/E6E7 cell-derived exosomes contained the HIV restriction miRNAs (miR-28, miR-29 family members, miR-125b, miR-150, miR-382, miR-223, miR-20a, and miR-198) (Fig. 6). It is known that miR-28, miR-125b, miR-150, miR-382, and miR-223 have the ability to bind to $3^{\prime}$ UTR of HIV transcripts through sequence complementarity and inhibit viral translation [47]. The miR-29 family members can target a highly conserved site in various HIV subtypes and suppress viral translation/replication [48]. Studies by several groups reported that miR-20a can competitively bind to p300/CREB-binding proteinassociated factor, an important cellular cofactor for the HIV Tat function, to inhibit HIV infection [48-50].

In summary, we demonstrated that HCEs could produce and release exosomes that contained the multiple antiviral factors. More importantly, the exosomes released by HCEs could be internalized by macrophages (Fig. 8). These findings suggest a novel anti-HIV mechanism through which HCEs as a bystander confer immune protection for HIV-infected macrophages. Because HIV has evolved several mechanisms to evade TLR3/IFN-me- diated intracellular innate immunity in the host cells, such as macrophages [51, 52], the participation in host anti-HIV activities by non-HIV target and bystander cells in the FRT is necessary and important for control of HIV infection through sexual transmission. The observation that exosomes produced/released by HCEs have the ability to transport multiple antiviral factors to the macrophages suggests an alternative and feasible strategy for exosome-based immunotherapy for HIV disease.

\section{Conflict of Interest Statement}

The authors declare no conflict of interest.

\section{Statement of Ethics}

Monocytes were obtained from Human Immunology Core at the University of Pennsylvania (Philadelphia, PA, USA). The core has the institutional review board approval for blood collection from healthy donors.

\section{Funding Sources}

This work was supported by the National Institutes of Health (DA041302, DA051396, and DA045568).

\section{Author Contributions}

X.-Q.X. and W.-Z.H. conceived and designed the experiments; X.-Q.X., Y.L., and L.G. performed the experiments; X.-Q.X. and B.Z. analyzed the data; F.-Z.M., W.-H.H., and X.W. contributed to reagents, materials, and analysis tools; X.-Q.X., W.-Z.H., and Adil Khan reviewed and revised the manuscript. All the authors have read, reviewed, and edited the manuscript and agreed for submission to this journal.

\section{References}

1 Hladik F, McElrath MJ. Setting the stage: host invasion by HIV. Nat Rev Immunol. 2008; $8(6): 447-57$.

2 Shattock RJ, Moore JP. Inhibiting sexual transmission of HIV-1 infection. Nat Rev Microbiol. 2003;1(1):25-34.

3 Zhang ZQ, Schuler T, Zupancic M, Wietgrefe S, Staskus KA, Reimann KA, et al. Sexual transmission and propagation of SIV and HIV in resting and activated CD4(+) T cells. Science. 1999;286:1353-7.

4 Iwasaki A. Innate immune recognition of HIV-1. Immunity. 2012;37(3):389-98.
5 Burgener A, McGowan I, Klatt NR. HIV and mucosal barrier interactions: consequences for transmission and pathogenesis. Curr Opin Immunol. 2015;36:22-30.

6 Wira CR, Fahey JV, Sentman CL, Pioli PA, Shen $\mathrm{L}$. Innate and adaptive immunity in $\mathrm{fe}$ male genital tract: cellular responses and interactions. Immunol Rev. 2005;206:306-35.

7 Nguyen PV, Kafka JK, Ferreira VH, Roth K, Kaushic C. Innate and adaptive immune responses in male and female reproductive tracts in homeostasis and following HIV infection. Cell Mol Immunol. 2014;11(5):41027.
8 Ank N, Paludan SR. Type III IFNs: new layers of complexity in innate antiviral immunity. Biofactors. 2009;35(1):82-7.

9 Fichorova RN, Cronin AO, Lien E, Anderson DJ, Ingalls RR. Response to Neisseria gonorrhoeae by cervicovaginal epithelial cells occurs in the absence of toll-like receptor 4-mediated signaling. J Immunol. 2002;168(5): 2424-32.

10 Zhou L, Li JL, Zhou Y, Liu JB, Zhuang K, Gao $\mathrm{JF}$, et al. Induction of interferon- $\lambda$ contributes to TLR3 and RIG-I activation-mediated inhibition of herpes simplex virus type 2 replication in human cervical epithelial cells. Mol Hum Reprod. 2015;21(12):917-29. 
11 Xu XQ, Guo L, Wang X, Liu Y, Liu H, Zhou $\mathrm{RH}$, et al. Human cervical epithelial cells release antiviral factors and inhibit hiv replication in macrophages. J Innate Immun. 2019; 11(1):29-40.

12 Lotvall J, Hill AF, Hochberg F, Buzas EI, Di Vizio D, Gardiner C, et al. Minimal experimental requirements for definition of extracellular vesicles and their functions: a position statement from the international society for extracellular vesicles. J Extracell Vesicles. 2014;3:26913.

13 Bang C, Thum T. Exosomes: new players in cell-cell communication. Int J Biochem Cell Biol. 2012;44(11):2060-4.

14 Zhang G, Yang P. A novel cell-cell communication mechanism in the nervous system: exosomes. J Neurosci Res. 2018;96(1):45-52.

15 Camussi G, Deregibus MC, Bruno S, Cantaluppi V, Biancone L. Exosomes/microvesicles as a mechanism of cell-to-cell communication. Kidney Int. 2010;78(9):838-48.

16 Schorey JS, Cheng Y, Singh PP, Smith VL. Exosomes and other extracellular vesicles in host-pathogen interactions. Embo Rep. 2015; 16(1):24-43.

17 Edgar JR. Q\&A: What are exosomes, exactly?. BMC Biol. 2016;14:46.

18 Stoorvogel W, Kleijmeer MJ, Geuze HJ, Raposo $\mathrm{G}$. The biogenesis and functions of exosomes. Traffic. 2002;3(5):321-30.

19 Simons M, Raposo G. Exosomes-vesicular carriers for intercellular communication. Curr Opin Cell Biol. 2009;21(4):575-81.

20 Mathivanan S, Ji H, Simpson RJ. Exosomes: extracellular organelles important in intercellular communication. J Proteomics. 2010; 73(10):1907-20.

21 Sun L, Wang X, Zhou Y, Zhou RH, Ho WZ, $\mathrm{Li}$ JL. Exosomes contribute to the transmission of anti-HIV activity from TLR3-activated brain microvascular endothelial cells to macrophages. Antiviral Res. 2016;134:16771.

22 Skotland T, Hessvik NP, Sandvig K, Llorente A. Thematic review series: exosomes and microvesicles: lipids as key components of their biogenesis and functions exosomal lipid composition and the role of ether lipids and phosphoinositides in exosome biology. J Lipid Res. 2019;60:9-18.

23 Madison MN, Roller RJ, Okeoma CM. Human semen contains exosomes with potent anti-HIV-1 activity. Retrovirology. 2014;11: 102.

24 Li J, Liu K, Liu Y, Xu Y, Zhang F, Yang H, et al. Exosomes mediate the cell-to-cell transmission of IFN- $\alpha$-induced antiviral activity. Nat Immunol. 2013;14(8):793-803.
25 Guo L, Xu X-Q, Zhou L, Zhou R-H, Wang X, $\mathrm{Li}$ J-L, et al. Human intestinal epithelial cells release antiviral factors that inhibit hiv infection of macrophages. Front Immunol. 2018; 9:247.

26 Sathe A, Reddy KV. TLR9 and RIG-I signaling in human endocervical epithelial cells modulates inflammatory responses of macrophages and dendritic cells in vitro. PLoS One. 2014;9(1):e83882.

27 Hijazi K, Cuppone AM, Smith K, Stincarelli MA, Ekeruche-Makinde J, De Falco G, et al. Expression of genes for drug transporters in the human female genital tract and modulatory effect of antiretroviral drugs. PLoS One. 2015;10(6): e0131405.

28 Li J, Wang Y, Wang X, Ye L, Zhou Y, Persidsky $Y$, et al. Immune activation of human brain microvascular endothelial cells inhibits HIV replication in macrophages. Blood. 2013; 121(15):2934-42.

29 Guo L, Xu XQ, Zhou L, Zhou RH, Wang X, Li $\mathrm{JL}$, et al. Human intestinal epithelial cells release antiviral factors that inhibit hiv infection of macrophages. Front Immunol. 2018;9:247.

30 Li J, Hu S, Zhou L, Ye L, Wang X, Ho J, et al. Interferon lambda inhibits herpes simplex virus type i infection of human astrocytes and neurons. Glia. 2011;59(1):58-67.

31 Wang X, Ye L, Hou W, Zhou Y, Wang YJ, Metzger DS, et al. Cellular microRNA expression correlates with susceptibility of monocytes/macrophages to HIV-1 infection. Blood. 2009;113(3):671-4.

32 Xing L, Wang S, Hu Q, Li J, Zeng Y. Comparison of three quantification methods for the TZM-bl pseudovirus assay for screening of anti-HIV-1 agents. J Virol Methods. 2016; 233:56-61.

33 Swaminathan G, Navas-Martín S, MartínGarcía J. MicroRNAs and HIV-1 infection: antiviral activities and beyond. J Mol Biol. 2014;426(6):1178-97.

34 Valadi H, Ekström K, Bossios A, Sjöstrand M, Lee JJ, Lötvall JO. Exosome-mediated transfer of mRNAs and microRNAs is a novel mechanism of genetic exchange between cells. Nat Cell Biol. 2007;9(6):654-9.

35 Abusamra AJ, Zhong ZH, Zheng XF, Li M, Ichim TE, Chin JL, et al. Tumor exosomes expressing fas ligand mediate CD8(+) T-cell apoptosis. BCMDFX. 2005;35:169-73.

36 Sampey GC, Meyering SS, Zadeh MA, Saifuddin M, Hakami RM, Kashanchi F. Exosomes and their role in CNS viral infections. J Neurovirol. 2014;20(3):199-208.

37 Janas T, Janas MM, Sapoń K, Janas T. Mechanisms of RNA loading into exosomes. Febs Lett. 2015;589(13):1391-8.

38 Hagiwara S, Kantharidis P, Cooper ME. MicroRNA as biomarkers and regulator of cardiovascular development and disease. Curr Pharm Des. 2014;20(14):2347-70.
39 Sadler AJ, Williams BR. Interferon-inducible antiviral effectors. Nat Rev Immunol. 2008; 8(7):559-68

40 Liu MQ, Zhou DJ, Wang X, Zhou W, Ye L, Li $J L$, et al. IFN- $\lambda 3$ inhibits HIV infection of macrophages through the JAK-STAT pathway. PLoS One. 2012;7(4):e35902.

41 Kunzi MS, Pitha PM. Role of interferon-stimulated gene ISG-15 in the interferon-omegamediated inhibition of human immunodeficiency virus replication. J Interferon Cytokine Res. 1996;16:919-27.

42 Okumura A, Lu G, Pitha-Rowe I, Pitha PM. Innate antiviral response targets HIV-1 release by the induction of ubiquitin-like protein ISG15. Proc Natl Acad Sci U S A. 2006; 103(5):1440-5.

43 Maitra RK, Silverman RH. Regulation of human immunodeficiency virus replication by 2',5'-oligoadenylate-dependent RNase L. J Virol. 1998;72(2):1146-52.

44 Liu Z, Pan Q, Ding S, Qian J, Xu F, Zhou J, et al. The interferon-inducible $\mathrm{MxB}$ protein inhibits HIV-1 infection. Cell Host Microbe. 2013;14(4):398-410.

45 Mihelich BL, Nonn L. Exosomal miRNAs contribute to prostatic zinc homeostasis. Cancer Res. 2012;72.

46 Mittelbrunn M, Gutiérrez-Vázquez C, Villarroya-Beltri C, González S, Sánchez-Cabo F, González MÁ, et al. Unidirectional transfer of microRNA-loaded exosomes from $\mathrm{T}$ cells to antigen-presenting cells. Nat Commun. 2011; 2:282.

47 Huang JL, Wang FX, Argyris E, Chen KY, Liang $\mathrm{ZH}$, Tian $\mathrm{H}$, et al. Cellular microRNAs contribute to HIV-1 latency in resting primary CD4(+) T lymphocytes. Nat Med. 2007;13: 1241-7.

48 Nathans R, Chu CY, Serquina AK, Lu CC, Cao H, Rana TM. Cellular microRNA and $\mathrm{P}$ bodies modulate host-HIV-1 interactions. Mol Cell. 2009;34(6):696-709.

49 Shapshak P. Molecule of the month HIV-1 Tat and miRNA. Bioinformation. 2012;8(23): $1123-4$

50 Triboulet R, Mari B, Lin YL, Chable-Bessia C, Bennasser Y, Lebrigand K, et al. Suppression of microRNA-silencing pathway by HIV-1 during virus replication. Science. 2007; 315(5818):1579-82.

51 Finlay BB, McFadden G. Anti-immunology: Evasion of the host immune system by bacterial and viral pathogens. Cell. 2006;124(4): 767-82.

52 Castiglione F, Bernaschi M. HIV-1 strategies of immune evasion. Int J Mod Phys C. 2005; 16(12):1869-78. 